\title{
HEAVY FERMION SUPERCONDUCTOR ORDER PARAMETER IN HIGH MAGNETIC FIELD
}

\author{
L. Kowalewski, R.J. Wojciechowski, M. Thomas and M.M. Nogala \\ Solid State Theory Division, Institute of Physics, A. Mickiewicz University \\ Matejki 48/49, 60-769 Poznań, Poland
}

It is shown that, independently on either the nature and the strength of pairing, the orbital effects of a high magnetic field in heavy fermion superconductor change the form of both the effective pairing and the gap function.

PACS numbers: 74.70.Tx, 74.60.-w, 74.90.+n

We consider the influence of a uniform high magnetic field $B$ on the superconducting order parameter of heavy fermion II-type superconductors at $T=0$.

The system is described in the slave boson mean field approach to the periodic Anderson Hamiltonian. We assume that the heavy fermion quasiparticles are caused by the $4 f$ electrons hybridization with conducting ones in the Landau states [1]. Moreover, the applied field is sufficiently high to take into account only the lowest occupied Landau level.

Thus we can start with the following effective Hamiltonian of the quasiparticles in strong magnetic field:

$$
H=H_{0}-V \int \mathrm{d} r \Psi_{\uparrow}^{+}(r) \Psi_{\downarrow}^{+}(r) \Psi_{\downarrow}(r) \Psi_{\uparrow}(r) .
$$

$H_{0}$ is the single quasiparticle Landau-type term, whereas the second one describes the local attractive quasiparticle interaction responsible for pairing.

In the discussed quantum limit (cf. [2]), when only the lowest Landau level is occupied, the appropriate Landau states take the form

$$
\varphi_{k}^{0}(r)=1 /\left(L_{x} L_{y}\right)^{1 / 2} \exp \left[\mathrm{i}\left(k y+k_{z} z\right)\right] \varphi_{\mathrm{osc}}^{0}\left(x-x_{0}\right) .
$$

Hereafter $k=\left(k, k_{z}\right)$, and the shifted harmonic oscillator function with guiding center $x_{0}=l^{2} k$ is given by

$$
\varphi_{\text {osc }}^{0}\left(x-x_{0}\right)=(2 a / \pi)^{1 / 4} \exp \left[-a\left(x-x_{0}\right)^{2}\right] \text {, }
$$

where the magnetic length $l=(\hbar / e B)^{1 / 2}$ and $a=1 / 2 l^{2}$.

In the normal phase the guiding oscillator centers of the free Landau quasiparticle states form an adequate close packed magnetic lattice. In the superconducting state we assume a square magnetic lattice whose quantum number $q$ is chosen so that the resulting magnetic structure contains one flux quantum $\phi_{0}=h c / 2 e$ per unit cell area $\pi l^{2}$ as in the low field case. Therefore $q=2 \pi / d$, where $d$ is a magnetic lattice period, and the reduced momenta $k \in(-q / 2, q / 2)[2,3]$. 
Since in the quantum limit the Landau-type functions have the Gaussian form restricted to the first magnetic Brillouin zone, the pairing occurs only within the magnetic unit lattice cell between reduced momenta $k$ with opposite sign.

The field operators $\Psi_{\sigma}(r)$ used in the Hamiltonian (1) can be now expanded in the set of quasiparticle eigenstates $\varphi_{k}(r)$ :

$$
\Psi_{\sigma}(r)=\sum_{k} d_{k \sigma} \varphi_{k}(r)
$$

$\sigma=\downarrow, \uparrow$. The latter are chosen as the superposition [3]:

$$
\varphi_{k}(r)=1 / N^{1 / 2} \sum_{n} \varphi_{k+n q}(r),
$$

where $q=(q, 0)$ and $N=e B L_{x} / \hbar q$.

Thus empolying (2) the Hamiltonian (1) can be written

$$
H=\sum_{k, \sigma} E_{k^{\prime}} d_{k \sigma}^{+} d_{k \sigma}-\sum_{k, k^{\prime}} V_{k k^{\prime}} d_{k \uparrow}^{+} d_{-k \downarrow}^{+} d_{-k_{\downarrow}^{\prime} \downarrow} d_{k^{\prime} \uparrow}
$$

The quasiparticle energies

$$
E_{k}=1 / 2\left\{\varepsilon_{k}+\left(\varepsilon_{f}+\lambda\right)-\left[\left(\varepsilon_{k}-\varepsilon_{f}\right)^{2}+4 \widetilde{W}^{2}\right]^{1 / 2}\right\}
$$

depend on the renormalized hybrydization parameter $\widetilde{W}$, on the energy shift $\lambda$ of the bare $4 f$-level position $\varepsilon_{f}$ which is defined relative to the equal to zero Fermi energy, and on the conduction electrons energies

$$
\varepsilon_{\boldsymbol{k}}=\hbar^{2} k_{z}^{2} / 2 m+\hbar \omega / 2
$$

where $\omega=e B / m$ is the cyclotron frequency. We neglect the Zeeman splitting in our analysis. The effective attractive pairing interaction in (3) takes the form

$$
V_{k k^{\prime}}=V_{k}^{(1)} V_{k^{\prime}}^{(1)}+V_{k}^{(2)} V_{k^{\prime}}^{(2)}
$$

and its terms are as follows:

$$
\begin{aligned}
& V_{k}^{(1)}=\left[V(a / \pi)^{1 / 2} L_{x} / N\right]^{1 / 2} \exp \left(-2 a l^{4} k^{2}\right), \\
& V_{k}^{(2)}=\left[V(a / \pi)^{1 / 2} L_{x} / N\right]^{1 / 2} \exp \left[-2 a l^{4}\left(k^{2}-q|k|\right)-a l^{4} q^{2} / 2\right],
\end{aligned}
$$

The heavy fermion superconductor order parameter $\Delta(r)$ may be obtained within the framework of de Gennes-Bogolyubov method [4]. Namely,

The quantity

$$
\Delta(r)=-V\left\langle\Psi_{\downarrow}(r) \Psi_{\uparrow}(r)\right\rangle=-V \sum_{k} \varphi_{k}(r) \varphi_{-k}(r)\left\langle d_{-k_{\downarrow}} d_{k \uparrow}\right\rangle .
$$

The correlation function at $T=0$ is found to be

$$
\left\langle d_{-k \downarrow} d_{k \uparrow}\right\rangle=-\frac{\Delta_{k}}{2 Q_{k}} .
$$

$$
Q_{k}=\left(E_{k}^{2}+\Delta_{k}^{2}\right)^{1 / 2}
$$


denotes the superconductor excited state energy and the energy gap function takes the form

$$
\Delta_{k}=V_{k}^{(1)} \Delta_{1}+V_{k}^{(2)} \Delta_{2}
$$

where

$$
\Delta_{1}=-\sum_{k^{\prime}} V_{k^{\prime}}^{(1)}\left\langle d_{-k^{\prime} \downarrow} d_{k^{\prime} \uparrow}\right\rangle, \quad \Delta_{2}=-\sum_{k^{\prime}} V_{k^{\prime}}^{(2)}\left\langle d_{-k^{\prime} \downarrow} d_{k^{\prime} \uparrow}\right\rangle .
$$

These two gap parameters are the solutions of the following self-consistent equations:

$$
\Delta_{1}=\Delta_{1} \sum_{k} \frac{\left(V_{k}^{(1)}\right)^{2}}{2 Q_{k}}+\Delta_{2} \sum_{k} \frac{V_{k}^{(1)} V_{k}^{(2)}}{2 Q_{k}}
$$

and

$$
\Delta_{2}=\Delta_{2} \sum_{k} \frac{\left(V_{k}^{(2)}\right)^{2}}{2 Q_{k}}+\Delta_{1} \sum_{k} \frac{V_{k}^{(1)} V_{k}^{(2)}}{2 Q_{k}}
$$

Finally, the considered order parameter has the form

$$
\begin{aligned}
\Delta(r) & =\left(V / N L_{y} L_{z}\right)(2 a / \pi)^{1 / 2} \sum_{k} \frac{V_{k}^{(1)} \Delta_{1}+V_{k}^{(2)} \Delta_{1}}{2 Q_{k}} \\
& \times\left(\sum_{n} \mathrm{e}^{\mathrm{i} n q y} \mathrm{e}^{-a\left[x-l^{2}(k+n q)\right]^{2}}\right)\left(\sum_{n^{\prime}} \mathrm{e}^{\mathrm{i} n^{\prime} q y} \mathrm{e}^{-a\left[x-l^{2}\left(k+n^{\prime} q\right)\right]^{2}}\right) .
\end{aligned}
$$

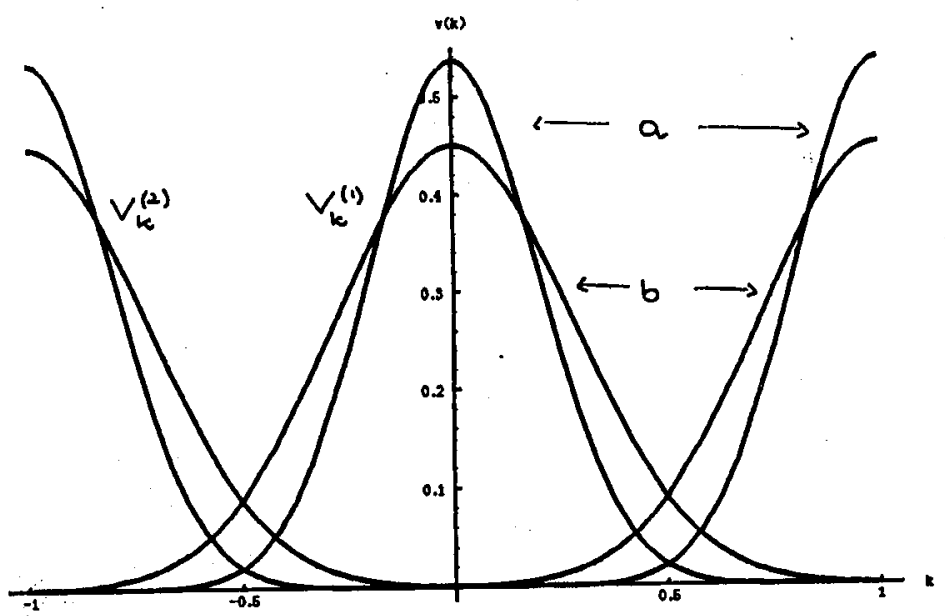

Fig. 1. The pairing interaction functions $V_{k}^{(1)}$ and $V_{k}^{(2)}$ within the magnetic Brillouin zone for (a) $5 \mathrm{~T}$ as well as (b) $10 \mathrm{~T}$ values of the magnetic field. 
As the first stage of numerical analysis we have examined the influence of the strength of a magnetic field on the interaction functions $V_{k}^{(1)}$ and $V_{k}^{(2)}$. We have realized (see Fig. 1) that with an increase of a magnetic field the role of $V_{k}^{(2)}$ becomes more important and it cannot be neglected if one would like to solve the obtained set of equations for $\Delta_{1}$ and $\Delta_{2}$.

We can conclude that the orbital, Landau-type redistribution of the quasiparticle states in the high magnetic field essentially changes the form of the effective pairing function independently on its physical nature and strength. Moreover, the orbital effects apparently modify the field dependence of the superconducting gap function in that case.

One can also expect a possible magnetic field dependence of the transition temperature. Numerical analysis of the latter as well as the gap function are in progress.

This work is supported by the Committee for Scientific Research under grant No. 2 P302 08904.

\section{References}

[1] R.J. Wojciechowski, L. Kowalewski, Physica B, 1993, in press.

[2] M. Rasolt, Z. Tešanović, Rev. Mod. Phys. 64, 709 (1992).

[3] V.N. Nicopoulos, P. Kumar, Phys. Rev. B 44, 12080 (1991).

[4] P.G. de Gennes, Superconductivity of Metals and Alloys, Addison-Wesley, Reading MA. 1989. 